\title{
Hsa_circ_0054633 regulates PDGF-BB-induced proliferation, migration and oxidative stress of vascular smooth muscle cells through miR-107/TXNIP axis
}

\section{Type}

Research paper

\section{Keywords}

Oxidative stress, miR-107, hsa_circ_0054633, TXNIP, HA-VSMCs

\begin{abstract}
Introduction

Hsa_circ_0054633 has been found to be elevated in the blood of coronary artery disease (CAD) patients. However, the molecular mechanism and the role of hsa_circ_0054633 in the pathogenesis of CAD have not been reported in detail.

\section{Material and methods}

The expression of hsa_circ_0054633, microRNA (miR)-107 and thioredoxin-interacting protein (TXNIP) mRNA was measured using quantitative real-time polymerase chain reaction. Human artery vascular smooth muscle cell (HA-VSMC) proliferation, cell cycle, and migration were detected by cell counting kit-8 assay, flow cytometry and transwell assay, respectively. The generation of reactive oxygen species (ROS) was analyzed by dichlorofluorescein diacetate (DCFH-DA) assay. Western blot was utilized to determine the levels of proliferating cell nuclear antigen (PCNA), cyclin D1, matrix metallopeptidase 9 (MMP-9), Mn-superoxide dismutase (SOD2) and TXNIP protein. The interaction between miR-107 and hsa_circ_0054633 or TXNIP was confirmed by dual-luciferase reporter, RNA immunoprecipitation assay or pull-down assay.
\end{abstract}

\section{Results}

Hsa_circ_0054633 was elevated in the plasma of CAD patients, and might be a potential blood biomarker for CAD prediction. Hsa_circ_0054633 silencing reversed PDGF-BB-induced promotion on HA-VSMC proliferation, cell cycle, migration and ROS production. MiR-107 directly interacted with hsa_circ_0054633 and TXNIP, and hsa_circ_0054633 regulated TXNIP expression by sponging miR-107. Besides, rescue assay indicated that the action of hsa_circ_0054633 silencing on PDGFBB-treated HA-VSMCs could be attenuated by miR-107 inhibition or TXNIP overexpression, respectively.

\section{Conclusions}

Hsa_circ_0054633 knockdown protected HA-VSMCs against PDGF-BB-induced dysfunction through regulating $\mathrm{miR}-107 / \mathrm{TXNIP}$ axis, suggesting a potential therapeutic target for coronary atherosclerosis. 
Hsa_circ_0054633 regulates PDGF-BB-induced proliferation, migration and oxidative stress of vascular smooth muscle cells through miR-107/TXNIP axis

Xiaochun Lei ${ }^{1}$, Yi Ding ${ }^{2}$, Ye Tian ${ }^{3}$, Peng Ding ${ }^{1 *}$

${ }^{1}$ Department of Cardiovascular Medicine, The Second Affiliated Hospital of Xi'an Medical University, Xi'an, Shaan’xi, China.

${ }^{2}$ Department of Nephrology and Endocrinology, The Second Affiliated Hospital of Xi'an Medical University, Xi'an, Shaan'xi, China.

${ }^{3}$ Department of Neurology, The Second Affiliated Hospital of Xi'an Medical University, Xi'an, Shaan'xi, China.

*Corresponding author: Peng Ding. Department of Cardiovascular Medicine, The

Second Affiliated Hospital of Xi'an Medical University, No. 167 Fangdong Street, Baqiao District 710038, Xi'an, Shaan’xi, China.

Tel: +86-15029002153Ｉ＿Email: zlqwr5@163.com

Running title: Hsa_circ_0054633 regulates PDGF-BB-induced dysfunction of HAVSMCs.

\section{Abstract}

Introduction: Hsa_circ_0054633 has been found to be elevated in the blood of coronary artery disease (CAD) patients. However, the molecular mechanism and the role of hsa_circ_0054633 in the pathogenesis of CAD have not been reported in detail. Materials and Methods: The expression of hsa_circ_0054633, microRNA (miR)-107 and thioredoxin-interacting protein (TXNIP) mRNA was measured using quantitative 
real-time polymerase chain reaction. Human artery vascular smooth muscle cell (HAVSMC) proliferation, cell cycle, and migration were detected by cell counting kit-8 assay, flow cytometry and transwell assay, respectively. The generation of reactive oxygen species (ROS) was analyzed by dichlorofluorescein diacetate (DCFH-DA) assay. Western blot was utilized to determine the levels of proliferating cell nuclear antigen (PCNA), cyclin D1, matrix metallopeptidase 9 (MMP-9), Mn-superoxide dismutase (SOD2) and TXNIP protein. The interaction between miR-107 and hsa_circ_0054633 or TXNIP was confirmed by dual-luciferase reporter, RNA immunoprecipitation assay or pull-down assay.

Results: Hsa_circ_0054633 was elevated in the plasma of CAD patients, and might be a potential blood biomarker for CAD prediction. Hsa_circ_0054633 silencing reversed PDGF-BB-induced promotion on HA-VSMC proliferation, cell cycle, migration and ROS production. MiR-107 directly interacted with hsa_circ_0054633 and TXNIP, and hsa_circ_0054633 regulated TXNIP expression by sponging miR-107. Besides, rescue assay indicated that the action of hsa_circ_0054633 silencing on PDGF-BB-treated HA-VSMCs could be attenuated by miR-107 inhibition or TXNIP overexpression, respectively.

Conclusion: Hsa_circ_0054633 knockdown protected HA-VSMCs against PDGF-BBinduced dysfunction through regulating miR-107/TXNIP axis, suggesting a potential therapeutic target for coronary atherosclerosis.

Keywords: hsa_circ_0054633, miR-107, TXNIP, oxidative stress, HA-VSMCs 


\section{Introduction}

Coronary artery disease (CAD) is a leading cause of mortality in the world. Despite great improvements in prevention, medical and interventional management, the incidence and mortality of $\mathrm{CAD}$ remain increasing in recent years [1, 2]. Atherosclerosis (AS) is a chronic inflammatory disease of the arterial wall, characterized by plaque formation, and is the well-recognized primary cause of CAD [3]. The pathogenesis of AS lesion formation is complex, and the initiating phase of AS plaques formation is the result of vascular smooth muscle cells (VSMCs) dysfunction [4]. Increasing researches have revealed VSMCs damage is associated with the regulation of atherosclerosis at tissue and molecular levels $[5,6]$. Thus, better understanding the molecular mechanism underlying abnormal VSMCs function is of great significance for the development of new therapeutics.

Circular RNAs (circRNAs) are a type of endogenous non-coding RNAs derived from reverse splicing of exons, introns or both $[7,8]$. They have covalently closed loop structures, which confer increasing stability relative to their linear transcripts [9]. Besides, many circRNAs are highly conserved and have tissue-specific expression patterns. Importantly, growing evidence has documented that circRNAs play significant roles in a variety of physiological and pathological processes, including cell cycle, proliferation, migration, invasion, tumorigenesis, immune responses and oxidative stress $[10,11]$. Thus, circRNAs are ideal candidates for future diagnostic biomarkers and therapeutic interventions [12]. Hsa_circ_0054633 is a novel identified circRNA. Zhao et al. demonstrated that hsa_circ_0054633 was up-regulated in the peripheral 
blood of type 2 diabetes mellitus (T2DM) patients, and was a potential blood biomarker for pre-diabetes and T2DM prediction [13]. Pan et al. revealed that hsa_circ_0054633 down-regulation enhanced high glucose-mediated inhibition on proliferation, migration and angiopoiesis of endothelial cells [14]. Importantly, Li et al. exhibited that hsa_circ_0054633 expression was up-regulated in the blood of CAD patients [15]. However, the role of hsa_circ_0054633 on VSMCs dysfunction remains unclear.

Emerging studies have reported that platelet-derived growth factor-BB (PDGF-BB) is one of the most potent stimulants for the dysfunction of VSMCs $[16,17]$. Thus, PDGF-BB was employed to induce VSMC dedifferentiation to mimic VSMC dysfunction in coronary atherosclerosis in vitro. This study aimed to explore the molecular mechanism and the role of hsa_circ_0054633 in PDGF-BB-induced VSMCs dysfunction, thus investigating the potential roles of hsa_circ_0054633 in the presence and progression of CAD.

\section{Materials and methods}

\section{Clinical samples}

A total of 33 patients with CAD were recruited from The Second Affiliated Hospital of Xi'an Medical University. There were 19 males and 14 females, ranging in age from 30 to 75 years. All CAD patients were diagnosed by coronary angiography in line with the guidelines established by American College of Cardiology/American Heart Association. The exclusion criteria were as follows: (1) cancer and other severe diseases; (2) serious infection within six weeks of the start of this work; (3) clinically acute or 
active chronic inflammatory disease. In the same period, a total of 33 healthy individuals were recruited to serve as the control group, and there were 18 females and 15 males with age ranging from 27 to 70 years.

Whole blood $(20 \mathrm{ml})$ was extracted from each participant on the day of admission to the study and transferred to anticoagulant tubes. Blood samples were centrifuged at $3000 \mathrm{~g}$ for $15 \mathrm{~min}$, and plasma was collected in a plastic tube, followed by centrifuging a second time at $3000 \mathrm{~g}$ for $15 \mathrm{~min}$ at room temperature. Subsequently, the plasma was collected and stored at $-80{ }^{\circ} \mathrm{C}$ before used. This study was approved by the Ethics Committee of The Second Affiliated Hospital of Xi'an Medical University and written informed consent was collected from all subjects.

\section{Cell culture and treatment}

Human artery vascular smooth muscle cells (HA-VSMCs) obtained from Chinese Academy of Sciences (Shanghai, China) were maintained in Dulbecco's modifed Eagle's medium (DMEM, Invitrogen, Waltham, MA, USA), which was supplemented with $10 \%$ fetal bovine serum (FBS) (Gibco, Carlsbad, CA, USA), at $37^{\circ} \mathrm{C}$ with $5 \%$ $\mathrm{CO}_{2}$. For the PDGF-BB group, HA-VSMCs were treated with $30 \mathrm{ng} / \mathrm{mL}$ PDGF-BB (Sigma, St. Louis, MO, USA) for $6 \mathrm{~h}$ at $37^{\circ} \mathrm{C}$.

\section{Quantitative real-time polymerase chain reaction (qRT-PCR)}

The extraction of total RNA from plasma and cultured HA-VSMCs was performed using TRIzol reagent (Invitrogen). First-strand complementary DNA (cDNA) was synthesized by using the Reverse Transcription System Kit (Takara, Dalian, China), then cDNA amplification was conducted using SYBR Green I (Takara) on the ABI7500 
111

112

system. The relative fold change in expression was assessed by $2^{-\Delta \Delta \mathrm{Ct}}$ method and normalized by glyceraldehyde-3-phosphate dehydrogenase (GAPDH) or U6 small nuclear B noncoding RNA (U6). The primers obtained from Qiagen (Valencia, CA, USA) were listed as followed: hsa_circ_0054633, F 5'$\begin{array}{lll}\text { TTGCTTTCTACACTTTCAGGTGAC-3' }{ }^{\prime} \text { and } & \text { R }\end{array}$ GCTTTTTGTCTGTAGTCAACCACCA-3'; miR-107, F 5'GTTAAGTCAGAGCGGGGCTT-3', and R 5'-CACTCCGCTTTTTCAGTGCC-3'; TXNIP, F 5'-GCCACACTTACCTTGCCAAT-3', and R 5'- TTGGATCCAGGAACG CTAAC-3'; GAPDH, F 5'-GTCAACGGATTTGGTCTGTATT-3', and R 5'AGTCTTCTGGGTGGCAGTGAT-3'; U6, F 5'-CTCGCTTCGGCAGCACA-3', and R 5'-AACGCTTCACGAATTTGCGT-3'.

\section{Cell transfection}

The mimic or inhibitor targeting miR-107 (miR-107 or anti-miR-107), and their corresponding negative control (miR-NC and anti-NC) were purchased from RIBOBIO (Guangzhou, China). Two forms of small interfering RNA (siRNA) were designed by Invitrogen to target hsa_circ_0054633 covalent closed junction (si-circ\#1, si-circ\#2), the same vector harboring a scrambled sequence was used as a negative control (si-NC). Also, pcDNA-TXNIP overexpression vector (TXNIP) and pcDNA negative control (vector) were synthesized by Invitrogen. Subsequently, the transfection was performed by Lipofectamine 2000 (Invitrogen).

\section{Cell counting kit-8 (CCK-8) assay}

After transfection and/or treatment, cells (5000/well) were cultivated into a 96-well 
133

134

plate overnight, and then interacted with $10 \mu \mathrm{L}$ CCK-8 solution (Dojindo Molecular Technologies, Japan) at $37^{\circ} \mathrm{C}$ for another $2 \mathrm{~h}$. Finally, the optical density of each well at $450 \mathrm{~nm}$ was analyzed by a microplate reader.

\section{Flow cytometry}

Following transfection and/or treatment, cells $\left(1 \times 10^{6}\right)$ were trypsinized and resuspended to obtain single-cell suspensions, then detached cells were fixed overnight in $70 \%$ ethanol, followed by staining with propidium iodide (Cell Cycle Detection kit; BD Biosciences, San Jose, CA, USA). Finally, quantitation of cell cycle distribution was analyzed with a FACScan flow cytometer (BD Biosciences). The percentage of the cells in $\mathrm{G} 0 / \mathrm{G} 1$, S, and G2/M phases was counted with FlowJo software.

\section{Transwell assay}

After transfection and/or treatment, cells in $200 \mathrm{~mL}$ serum-free DMEM were seeded into the upper chamber of a transwell insert (Cell Biolabs, Inc. Santiago, CA, USA), then lower chamber was filled with $600 \mathrm{~mL}$ medium supplementing with $10 \%$ FBS. Following incubation at $37^{\circ} \mathrm{C}$ with $5 \% \mathrm{CO}_{2}$ for $24 \mathrm{~h}$, cells on the lower face of the membranes were fixed and stained. Finally, migrated cells from 10 randomly selected fields were counted by a microscope.

\section{Detection of reactive oxygen species (ROS)}

The production of ROS was measured by Dichlorofluorescein diacetate (DCFHDA) assay. Transfected and/or treated HA-VSMCs were suspended using $20 \mathrm{mM} 2^{\prime}, 7^{\prime}-$ Dichlorodihydrofluorescein diacetate (DCFH-DA; Sigma) for $30 \mathrm{~min}$ at $37^{\circ} \mathrm{C}$ in the dark. After washing with PBS three times, the fluorescence intensity of the samples was 
detected using a fluorescence microplate reader at $488 \mathrm{~nm}$ excitation wavelength and $525 \mathrm{~nm}$ emission wavelength, respectively.

\section{Western blot}

Total protein was extracted using RIPA buffer (Beyotime, Shanghai, China) from cells, and protein concentrations were determined using the bicinchoninic acid (BCA) Protein Assay kit (Takara). Protein extractions were separated by 12\% sodium lauryl sulfate-polyacrylamide gels (SDS-PAGE) and transferred to polyvinylidene difluoride membranes (PVDF; Millipore, Billerica, MA, USA). Then membranes were interacted with primary antibodies against proliferating cell nuclear antigen (PCNA) (1:5000, ab29), cyclin D1 (1:10000, ab134175), matrix metallopeptidase 9 (MMP-9) (1:2000, ab38898), Mn-superoxide dismutase (SOD2) (1:5000, ab13533), TXNIP (1:2000, ab188865) (Abcam, Cambridge, MA, USA), and the secondary antibody anti-rabbit IgG-horseradish peroxidase at a 1:1000 dilution (Sangon, Shanghai, China). $\beta$-actin (1:2000, 4967, Cell Signaling Technology, Boston, MA, USA) was used as an internal control.

\section{Dual-luciferase reporter assay}

The sequences of TXNIP 3'-UTR or hsa_circ_0054633 containing the wild-type (wt) or mutant (mut) potential binding sites of miR-107 were amplified and subcloned into pRL-TK luciferase reporter vector (Promega, Madison, WI, USA), respectively. Then cells were placed in 24-well plates and co-transfected with $10 \mathrm{ng}$ wt or mut constructed luciferase vectors, and $40 \mathrm{nM}$ of miR-107 mimic or miR-NC mimic. The luciferase activity was measured by a dual luciferase assay kit (Promega) and 
normalized by renilla luciferase activity.

\section{RNA immunoprecipitation (RIP) assay}

HA-VSMCs were lysed with RIP lysis buffer (Millipore, Billerica, MA, USA). Cell lysis was co-immunoprecipitated with magnetic beads containing human anti-Ago2 antibody (Abcam). Normal mouse IgG (Millipore) was used as a negative control. Finally, immunoprecipitated RNA was purified and subjected to qRT-PCR analysis.

\section{Pull-down assay}

Biotin (Bio)-miR-107 and Bio-NC were synthesized by GenePharma Company (Shanghai, China), and then transfected into HA-VSMCs for $48 \mathrm{~h}$. Subsequently, cells were lysed, and the extracts were incubated with streptavidin-coated magnetic beads (Invitrogen). After elution, the bead-bound RNA complex was isolated and subjected for reverse transcription using qRT-PCR analysis.

\section{Statistical analysis}

All experiments were performed three times. Data were expressed as mean \pm standard deviation (SD). Statistical differences in different groups were analyzed by Student's $t$ test or one-way analysis of variance (ANOVA) with GraphPad Prism 7 software. Receiver operating characteristic (ROC) curves were plotted to analyze the diagnostic value of hsa_circ_0054633. $P<0.05$ indicated statistically significant.

\section{Results}

\section{Demographic characteristics of CAD patients and controls}

In this study, the demographic data, laboratory parameters of CAD patients and 
controls were summarized in Table 1. As expected, there were significant differences in hypertension, total cholesterol, triglyceride, LDL-cholestero (LDL-C), and highsensitivity C-reactive protein (Hs-CRP) between these two subgroups $(P<0.01)$. However, other factors, including sex, age, body mass index, cigarette smoking, drinking, diabetes mellitus, and HDL-C, were not significantly different $(P>0.05)$.

\section{Hsa_circ_0054633 expression is elevated in CAD patients and PDGF-BB-induced}

\section{HA-VSMCs}

The expression of hsa_circ_0054633 was detected and results showed hsa_circ_0054633 expression was up-regulated in the plasma of CAD patients relative to the plasma of healthy people (Fig. 1A). Moreover, ROC curves revealed that the area under the ROC curve (AUC) was 0.762 (95\% confidence interval (CI): 0.637-0.887), the cut-off value was 1.615 , and the sensitivity and specificity were $63.64 \%$ and $96.97 \%$, respectively (Fig. 1B). Furthermore, highly expressed hsa_circ_0054633 were associated with smoking history, with symptoms of hypertension and diabetes mellitus, and with total cholesterol $\geqslant 5.1 \mathrm{mmol} / \mathrm{L}$ and triglyceride $\geqslant 1.8 \mathrm{mmol} / \mathrm{L}(P<0.05$; Table 2). Besides that, hsa_circ_0054633 expression was also up-regulated in PDGFBB-induced HA-VSMCs compared with the cells untreated with PDGF-BB (Fig. 1C). .

\section{Hsa_circ_0054633 knockdown reverses PDGF-BB-induced HA-VSMCs}

\section{dysfunction}

To investigate the role of hsa_circ_0054633 in CAD, we knocked down hsa_circ_0054633 in HA-VSMCs by transfecting with two forms of constructed sihsa_circ_0054633 plasmid (si-circ\#1, si-circ\#2). Then the effects of si-circ\#1 and si- 
circ\#2 were determined, and qRT-PCR analysis showed both si-circ\#1 and si-circ\#2 remarkably declined hsa_circ_0054633 expression compared with the si-NC group (Fig. 2A). Subsequently, HA-VSMCs were treated with PDGF-BB, PDGF-BB + si-NC, or PDGF-BB + si-circ\#1 to assay cell cycle, proliferation, migration and ROS production, and we found hsa_circ_0054633 silence overturned PDGF-BB-mediated enhancement of hsa_circ_0054633 expression (Fig. 2B). After that, CCK-8 assay indicated hsa_circ_0054633 knockdown attenuated PDGF-BB-induced promotion on HAVSMC proliferation (Fig. 2C). Flow cytometric analysis showed PDGF-BB reduced the number of HA-VSMCs in the G0/G1 phase and increased the number of HAVSMCs in the S phase, suggesting PDGF-BB promoted cell cycle progression, while this promotion was abated by hsa_circ_0054633 knockdown(Fig. 2D). Transwell assay exhibited that the increase of migrated HA-VSMCs induced by PDGF-BB was mitigated by the silencing of hsa_circ_0054633 (Fig. 2E). Besides that, we found PDGF-BB induced the generation of ROS, while hsa_circ_0054633 knockdown inhibited the production of ROS in HA-VSMCs (Fig. 2F). In addition, western blot showed hsa_circ_0054633 down-regulation also decreased PDGF-BB-mediated upregulation of PCNA, cyclin D1, MMP9, and SOD2 (Fig. 2G), further indicating hsa_circ_0054633 knockdown reversed PDGF-BB-induced proliferation, cell cycle, migration and oxidative damage in HA-VSMCs.

\section{Hsa_circ_0054633 is a sponge of miR-107}

The molecular mechanisms underlying the action of hsa_circ_0054633 on HAVSMC properties were explored. According to the prediction of starBase online 
243

244

program, we found miR-107 had the potential binding sites of hsa_circ_0054633 (Fig. 3A). The expression of miR-107 was detected and results showed miR-107 expression was down-regulated in the plasma of CAD patients compared with the plasma from healthy people (Fig. 3B), moreover, lowly expressed miR-107 was related to smoking history, symptoms of hypertension, total cholesterol $\geqslant 5.1 \mathrm{mmol} / \mathrm{L}$ and triglyceride $\geqslant 1.8 \mathrm{mmol} / \mathrm{L} \quad(P<0.05$; Table 2$)$. Also, PDGF-BB decreased the expression of miR-107 in HA-VSMCs (Fig. 3C). Thus, miR-107 might be a potential biomarker for the development of CAD. Subsequently, we verified miR-107 mimic transfection could up-regulate miR-107 expression in HA-VSMCs relative to miR-NC mimic transfection (Fig. 3D). Then the interaction between hsa_circ_0054633 and miR-107 was analyzed. The dual luciferase reporter assay showed miR-107 mimic reduced the luciferase activity in HA-VSMCs cells transfected with the hsa_circ_0054633-wt (circ-wt) (Fig. 3E). RIP assay demonstrated that hsa_circ_0054633 and miR-107 were enriched in Ago2 immunoprecipitates compared with control IgG immunoprecipitates (Fig. 3F). Moreover, HA-VSMCs transfected with the bio-miR-107 showed elevated hsa_circ_0054633 levels but exhibited no changes in bio-NC (Fig. 3G). Besides that, we also observed that hsa_circ_0054633 knockdown up-regulated miR-107 expression in HA-VSMCs (Fig. 3H). Taken together, hsa_circ_0054633 was a sponge of miR-107 and negatively regulated its expression.

\section{Hsa_circ_0054633 knockdown alleviates PDGF-BB-induced HA-VSMCs} dysfunction via targeting miR-107

We further studied whether miR-107 involved in hsa_circ_0054633-mediated HA- 
VSMC dysfunction. First, HA-VSMCs were transfected with anti-NC or anti-miR-107, and anti-miR-107 transfection significantly reduced the level of miR-107 (Fig. 4A). Next, HA-VSMCs were transfected with si-NC, si-circ\#1, si-circ\#1 + anti-NC, or sicirc\#1 + anti-miR-107 after treatment with PDGF-BB, and qRT-PCR analysis showed hsa_circ_0054633 knockdown elevated miR-107 expression, while this elevation was reduced by miR-107 inhibition (Fig. 4B). After that, functional experiments were conducted and results exhibited that miR-107 inhibition reversed hsa_circ_0054633 knockdown-induced reduction of the number of migratory (Fig. 4C) and proliferating cell (Fig. 4D), cell cycle arrest (Fig. 4E), and ROS production (Fig. 4F) in PDGF-BBtreated HA-VSMCs. Western blot analysis displayed that hsa_circ_0054633 downregulation also decreased PDGF-BB-mediated up-regulation of PCNA, cyclin D1, MMP9, and SOD2, while these down-regulations were rescued by miR-107 inhibition in HA-VSMCs (Fig. 4G). Altogether, hsa_circ_0054633 alleviated PDGF-BB-induced HA-VSMC dysfunction by targeting miR-107.

\section{TXNIP is a target of miR-107}

Through searching the starBase program, we found TXNIP contained the binding sites of miR-107 (Fig. 5A). TXNIP expression was found to be up-regulated in the plasma from CAD patients relative to the plasma from healthy people (Fig. 5B), and high expression of TXNIP was linked with symptoms of hypertension, and total cholesterol $\geqslant 5.1 \mathrm{mmol} / \mathrm{L}$ as well as triglyceride $\geqslant 1.8 \mathrm{mmol} / \mathrm{L}(P<0.05$; Table 2$)$, suggesting TXNIP increase might be associated with the development of CAD. Similarly, TXNIP expression at mRNA and protein levels also increased in PDGF-BB- 
induced HA-VSMCs (Fig. 5C, D). Afterwards, a decline of luciferase activity in HAVSMCs cells co-transfected with TXNIP 3'UTR-wt and miR-107 mimic confirmed the direct interaction between TXNIP and miR-107 (Fig. 5E). Subsequent western bolt analysis displayed that miR-107 overexpression decreased the level of TXNIP (Fig. 5F, G). These data verified that miR-107 targetedly suppressed TXNIP expression. Importantly, we also observed that hsa_circ_0054633 silence decreased the level of TXNIP in HA-VSMCs, while this decrease was rescued by miR-107 inhibition (Fig. 5H, I), thus hsa_circ_0054633 could indirectly regulate TXNIP expression through miR-107.

\section{Hsa_circ_0054633 moderates PDGF-BB-induced HA-VSMCs damage by} regulating TXNIP

Given the relationship between hsa_circ_0054633 and TXNIP, we further investigated whether TXNIP participated in the action of hsa_circ_0054633 on HAVSMCs. First, HA-VSMCs were transfected with TXNIP or vector, by contrast to vector transfection, TXNIP transfection significantly elevated the expression of TXNIP at mRNA and protein levels (Fig. 6A, B). Then HA-VSMCs were transfected with siNC, si-circ\#1, si-circ\#1 + vector, or si-circ\#1 + TXNIP after treatment with PDGF-BB, we found TXNIP rescued hsa_circ_0054633 knockdown-induced down-regulation of TXNIP expression (Fig. 6C, D), further indicating hsa_circ_0054633 could regulate TXNIP expression. Subsequently, rescued assay showed TXNIP up-regulation reversed hsa_circ_0054633 knockdown-induced migration suppression (Fig. 6E), decreased ROS production (Fig. 6F), and proliferation inhibition (Fig. 6G) and cell cycle arrest 
309

310

(Fig. 6H) in PDGF-BB-treated HA-VSMCs. Besides that, TXNIP up-regulation also elevated the levels of PCNA, cyclin D1, MMP9, and SOD2 mediated by hsa_circ_0054633 knockdown in PDGF-BB-treated HA-VSMCs (Fig. 6I). In all, hsa_circ_0054633 moderated PDGF-BB-induced HA-VSMC dysfunction by regulating TXNIP.

\section{Discussion}

VSMC phenotypic switching is an early event in atherosclerosis and neointimal formation, and the involvement of circRNAs in the regulation of phenotypic switching

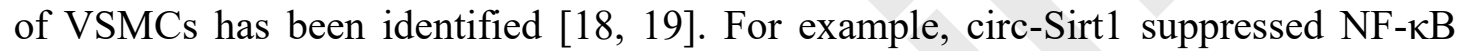
activation by up-regulating SIRT1 expression via sponging miR-132/212 to hinder inflammatory phenotypic switching of VSMCs [20]. Circ-SATB2 promoted VSMC phenotypic proliferation, apoptosis, differentiation and migration through up-regulating STIM1 expression [21]. CircRNA_0020397 enhanced VSMC proliferation via regulating miR-138/KDR axis to alleviate intracranial aneurysm progression [22]. Circ_RUSC2 overexpression affected phenotypic modulation of VSMCs by contributing to proliferation, migration and suppressing apoptosis through increasing miR-661-mediated SYK expression [23]. Thus, circRNAs may be important factors in the dysfunction of VSMCs. In this study, hsa_circ_0054633 expression was higher in the plasma of CAD patients than that in healthy individuals, besides, hsa_circ_0054633 showed a higher ROC AUC, and might be a potential diagnostic biomarker for early prediction of CAD. Additionally, hsa_circ_0054633 expression was also up-regulated 
in PDGF-BB-induced cells, suggesting that hsa_circ_0054633 elevation might be associated with the development of CAD. Then functional experiments showed hsa_circ_0054633 silence reversed PDGF-BB-induced promotion on HA-VSMC proliferation, cell cycle, and migration, thereby impeding the formation of atherosclerosis. PCNA is a gene on chromosome 20pter-p12 that encodes a homotrimeric nuclear protein that acts as a processivity factor for DNA polymerase delta in eukaryotic cells and is essential for DNA replication, repair, and recombination [24]. Cyclin D1 (CCND1) is a proto-oncogene located on chromosome 11q13 that is an essential regulator of the G1-S transition in cell cycle control progression [25]. MMP9 is a matrixin, a class of enzymes that belong to the zinc-metalloproteinases family involved in the degradation of collagens IV and V, gelatins I and V, and fibronectin, and plays a important role in local proteolysis of the extracellular matrix and in leukocyte migration [26]. In this study, western blot analysis indicated hsa_circ_0054633 downregulation also decreased PDGF-BB-mediated up-regulation of PCNA, cyclin D1 and MMP9 expression, further revealing the involvement of hsa_circ_0054633 in PDGFBB-induced HA-VSMC dysfunction. ROS always is generated during the inflammatory response, which oxidizes low-density lipoproteins and results in structural damage and dysfunction of endothelial cells, ultimately leads to vascular remodeling [27-29]. Also, the antioxidant system, such as SOD2 enzyme, a mitochondrial enzyme that catalyzes the conversion of $\mathrm{O}^{--}$to hydrogen peroxide (H2O2), and the amount of ROS are kept in a certain state of homeostasis [30-32]. In this study, we also found hsa_circ_0054633 silence reversed PDGF-BB-induced ROS 
353

production and SOD2 elevation in HA-VSMCs. Thus, hsa_circ_0054633 was important in VSMC homeostasis.

MicroRNAs (miRNAs) are a class of small noncoding RNA molecules, which have been revealed to play important roles in multiple cellular processes, including cell proliferation, apoptosis, migration, tumorigenesis, oxidative stress and differentiation [33-35]. Increasing evidence suggests that many miRNAs involve in VSMC phenotypic modulation [36]. MiR-107 is a functional miRNA. Gao et al. revealed that miR-107 overexpression reduced the level of blood lipid and atherosclerotic index, suppressed vascular endothelial cell (VEC) apoptosis, inflammation and endoplasmic reticulum stress through KRT1-dependent Notch signaling pathway in coronary atherosclerosis [37]. Besides that, miR-107 was found to be decreased in the blood of AS patients, and involved in circRNA-0044073-mediated promotion of the proliferation and invasion of human VSMCs and human VECs [38]. In this study, miR107 was confirmed to be a target of hsa_circ_0054633. MiR-107 was decreased in HAVSMCs, and was down-regulated by PDGF-BB and hsa_circ_0054633. What's more, miR-107 inhibition reversed hsa_circ_0054633 silence-mediated inhibition of the proliferation, cell cycle, migration and ROS generation in PDGF-BB-induced HAVSMCs.

TXNIP is an endogenous inhibitor and regulator of thioredoxin (TRX), and modulate oxidative stress via repressing antioxidant activity of TRX [39]. TXNIP is abundant in the vascular wall [40], and involves in controlling vascular neointimal lesion formation [41]. TXNIP promotes Ox-LDL exposure-induced inflammatory 
injuries of human aortic endothelial cells (HAECs) to contribute to atherosclerotic development [42]. This study found TXNIP was up-regulated in the plasma of CAD patients and PDGF-BB-induced HA-VSMCs. TXNIP was a target of miR-107, and hsa_circ_0054633 served as a competing endogenous RNA for miR-107 to regulate TXNIP expression in HA-VSMCs. Additionally, TXNIP overexpression overturned the inhibitory action of hsa_circ_0054633 knockdown on PDGF-BB-induced HA-VSMC dysfunction.

However, although some interesting results have been drawn from our study, there are still some limitations. First, we mainly performed our research work in PDGF-BBinduced HA-VSMC. Further researches should be carried out in vivo and a larger cohort of the disease. People may put efforts in making use of animal models with high or low hsa_circ_0054633 expression in mice in the future. Besides that, the involvement of circRNAs in regulating cell functions is very complex, new study should be conducted to explore other potential molecular mechanisms underlying hsa_circ_0054633 in HAVSMC dysfunction.

In conclusion, this study demonstrated that hsa_circ_0054633 knockdown protected HA-VSMCs from PDGF-BB-induced dysfunction through up-regulating miR-107 and subsequent down-regulating TXNIP expression, suggesting a useful strategy for coronary atherosclerosis therapy. 
400

401

402

403

404

405

406

407

408

409

410

411

412

413

414

415

416

417

418

\section{Acknowledgements}

Not applicable.

\section{Funding}

No funding was received.

\section{Availability of data and materials}

The analyzed data sets generated during the present study are available from the corresponding author on reasonable request.

\section{Authors' contribution}

Conceptualization, Methodology, Formal analysis and Data curation: Yi Ding and Ye Tian; Validation and Investigation: Xiaochun Lei and Peng Ding; Writing - original draft preparation and Writing - review and editing: Xiaochun Lei, Yi Ding and Peng Ding; Approval of final manuscript: all authors

\section{Ethics approval and consent to participate}

The present study was approved by the ethical review committee of the Second Affiliated Hospital of Xi'an Medical University.

Not applicable. 


\section{Competing interests}

421 The authors declare that they have no competing interests.

\section{References}

423 1. Abubakar I, Tillmann T, Banerjee A. Global, regional, and national age-sex specific all-cause and cause-specific mortality for 240 causes of death, 1990-2013: a systematic analysis for the Global Burden of Disease Study 2013. Lancet 2015; Microparticles and their role in coronary artery disease. Int J Cardiol 2017; 230:

3. Lusis A J. Atherosclerosis. Nature 2000; 407: 233-41.

4. Johnson J L. Emerging regulators of vascular smooth muscle cell function in the development and progression of atherosclerosis. Cardiovasc Res 2014; 103: 45260.

5. Hutcheson J D, Goettsch C, Bertazzo S, et al. Genesis and growth of extracellularvesicle-derived microcalcification in atherosclerotic plaques. Nat Mater 2016; 15 : 335.

6. Qu Y, Zhang N. miR-365b-3p inhibits the cell proliferation and migration of human coronary artery smooth muscle cells by directly targeting ADAMTS1 in coronary atherosclerosis. Exp Ther Med 2018; 16: 4239-45. 
an exon-containing lariat precursor. Elife 2015; 4: e07540.

442

443

444

445

446

447

448

449

450

451

452

453

454

455

456

457

458

459

460

461

462

8. Li Z, Huang C, Bao C, et al. Exon-intron circular RNAs regulate transcription in the nucleus. Nat Struct Mol Biol 2015; 22: 256.

9. Ebbesen K K, Kjems J, Hansen T B. Circular RNAs: identification, biogenesis and function. Biochim Biophys Acta 2016; 1859: 163-8.

10. Panda A C, Abdelmohsen K, Gorospe M. SASP regulation by noncoding RNA. Mech Ageing Dev 2017; 168: 37-43.

11. Hombach S, Kretz M. Non-coding RNAs: classification, biology and functioning. Adv Exp Med Biol 2016; 937: 3-17.

12. Ma H, Xu Y, Zhang R, Guo B, Zhang S, Zhang X. Differential expression study of circular RNAs in exosomes from serum and urine in patients with idiopathic membranous nephropathy. Arch Med Sci 2019; 15:738-753.

13. Zhao Z, Li X, Jian D, Hao P, Rao L, Li M. Hsa_circ_0054633 in peripheral blood can be used as a diagnostic biomarker of pre-diabetes and type 2 diabetes mellitus. Acta Diabetol 2017; 54: 237-45.

14. Pan L, Lian W, Zhang X, et al. Human circular RNA0054633 regulates high glucoseinduced vascular endothelial cell dysfunction through the microRNA218/roundabout 1 and microRNA218/heme oxygenase 1 axes. Int J Mol Med 2018; 42: 597-606.

15. Li X, Zhao Z, Jian D, Li W, Tang H, Li M. Hsa-circRNA11783-2 in peripheral blood is correlated with coronary artery disease and type 2 diabetes mellitus. Diab Vasc Dis Res 2017; 14: 510-5. 
16. Shawky N M, Segar L. Sulforaphane inhibits platelet-derived growth factorinduced vascular smooth muscle cell proliferation by targeting mTOR/p70S6kinase signaling independent of Nrf2 activation. Pharmacol Res 2017; 119: 251-64.

17. Millette E, Rauch B H, Kenagy R D, Daum G, Clowes A W. Platelet-derived growth factor-BB transactivates the fibroblast growth factor receptor to induce proliferation in human smooth muscle cells. Trends Cardiovasc Med 2006; 16: 258.

18. Ballantyne M D, Pinel K, Dakin R, et al. Smooth Muscle Enriched Long Noncoding RNA (SMILR) Regulates Cell Proliferation. Circulation 2016; 133: 2050-65.

19. Iaconetti C, De Rosa S, Polimeni A, et al. Down-regulation of miR-23b induces phenotypic switching of vascular smooth muscle cells in vitro and in vivo. Cardiovasc Res 2015; 107: 522-33.

20. Kong P, Yu Y, Wang L, et al. circ-Sirt1 controls NF-kappaB activation via sequence-specific interaction and enhancement of SIRT1 expression by binding to miR-132/212 in vascular smooth muscle cells. Nucleic Acids Res 2019; 47: 358093.

21. Mao Y Y, Wang J Q, Guo X X, Bi Y, Wang C X. Circ-SATB2 upregulates STIM1 expression and regulates vascular smooth muscle cell proliferation and differentiation through miR-939. Biochem Biophys Res Commun 2018; 505: 11925. 
22. Wang Y, Wang Y, Li Y, et al. Decreased expression of circ_0020397 in intracranial aneurysms may be contributing to decreased vascular smooth muscle cell proliferation via increased expression of miR-138 and subsequent decreased KDR expression. Cell Adh Migr 2019; 13: 220-8.

23. Sun J, Zhang Z, Yang S. Circ_RUSC2 upregulates the expression of miR-661 target gene SYK and regulates the function of vascular smooth muscle cells. Biochem Cell Biol 2019; 97: 709-14.

24. Park SY, Jeong MS, Han CW, Yu HS, Jang SB. Structural and Functional Insight into Proliferating Cell Nuclear Antigen. J Microbiol Biotechnol 2016; 26:637-647.

25. John RR, Malathi N, Ravindran C, Anandan S. Mini review: Multifaceted role played by cyclin D1 in tumor behavior. Indian J Dent Res 2017; 28:187-192.

26. Makarenkova HP, Meech R. Barx homeobox family in muscle development and regeneration. Int Rev Cell Mol Biol 2012; 297:117-173.

27. Zhang Y, Huang J, Yang X, et al. Altered Expression of TXNIP in the peripheral leukocytes of patients with coronary atherosclerotic heart disease. Medicine 2017; 96: e9108.

28. Derosa G, Maffioli P, D’Angelo A, Russo R. Effects of a nutraceutical combination of monacolin, $\gamma$-oryzanol and $\gamma$-aminobutyric acid on lipid profile and C-reactive protein in mice. Arch Med Sci 2019, 15:792.

29. Pavlović I, Pejić S, Radojević-Škodrić S, et al. The effect of antioxidant status on overall survival in renal cell carcinoma. Arch Med Sci 2020, 16:94.

30. Chang D, Zhang $\mathrm{X}$, Rong $\mathrm{S}$, et al. Serum antioxidative enzymes levels and 
oxidative stress products in age-related cataract patients. Oxid Med Cell Longev $2013 ; 2013: 587826$.

31. Kaczmarczyk-Sedlak I, Folwarczna J, Sedlak L, et al. Effect of caffeine on biomarkers of oxidative stress in lenses of rats with streptozotocin-induced diabetes. Arch Med Sci 2019, 15:1073.

32. Klisic A, Kavaric N, Stanisic V, et al. Endocan and a novel score for dyslipidemia, oxidative stress and inflammation (DOI score) are independently correlated with glycated hemoglobin $(\mathrm{HbA} 1 \mathrm{c})$ in patients with prediabetes and type 2 diabetes. Arch Med Sci 2020, 16:42.

33. Cheng A M, Byrom M W, Shelton J, Ford L P. Antisense inhibition of human miRNAs and indications for an involvement of miRNA in cell growth and apoptosis. Nucleic Acids Res 2005; 33: 1290-7.

34. Chen C-Z, Li L, Lodish H F, Bartel D P. MicroRNAs modulate hematopoietic lineage differentiation. Science 2004; 303: 83-6.

35. Banerjee J, Khanna S, Bhattacharya A. MicroRNA Regulation of Oxidative Stress. Oxid Med Cell Longev 2017; 2017: 2872156.

36. Guo X, Li D, Chen M, et al. miRNA-145 inhibits VSMC proliferation by targeting CD40. Sci Rep 2016; 6: 35302.

37. Gao Z F, Ji X L, Gu J, Wang X Y, Ding L, Zhang H. microRNA - 107 protects against inflammation and endoplasmic reticulum stress of vascular endothelial cells via KRT1 - dependent Notch signaling pathway in a mouse model of coronary atherosclerosis. J Cell Physiol 2019; 234: 12029-41. 
38. Shen L, Hu Y, Lou J, et al. CircRNA-0044073 is upregulated in atherosclerosis and increases the proliferation and invasion of cells by targeting miR-107. Mol Med Rep 2019; 19: 3923-32.

39. Devi T S, Hosoya K-I, Terasaki T, Singh L P. Critical role of TXNIP in oxidative stress, DNA damage and retinal pericyte apoptosis under high glucose: implications for diabetic retinopathy. Exp Cell Res 2013; 319: 1001-12.

40. Berk B C. Novel approaches to treat oxidative stress and cardiovascular diseases. Trans Am Clin Climatol Assoc 2007; 118: 209.

41. Perrone L, Devi T S, Hosoya K i, Terasaki T, Singh L P. Thioredoxin interacting protein (TXNIP) induces inflammation through chromatin modification in retinal capillary endothelial cells under diabetic conditions. J Cell Physiol 2009; 221: 262-72.

42. Chen M, Li W, Zhang Y, Yang J. MicroRNA-20a protects human aortic endothelial cells from Ox-LDL-induced inflammation through targeting TLR4 and TXNIP signaling. Biomed Pharmacother 2018; 103: 191-7.

\section{Figure legends}

\section{Fig. 1 Hsa_circ_0054633 expression is elevated in CAD patients and PDGF-BB-} induced HA-VSMCs. (A) The expression of hsa_circ_0054633 was detected in the plasma from CAD patients and healthy people using qRT-PCR. (B) ROC curves were plotted to determine the diagnostic value of hsa_circ_0054633 in plasma of CAD 
551

552

553

554

555

556

557

558

559

560

561

562

563

564

565

566

567

568

569

570

571

572

patients. (C) The expression of hsa_circ_0054633 was measured in HA-VSMCs treated with or without PDGF-BB by qRT-PCR. ${ }^{*} P<0.05, * * P<0.01$.

Fig. 2 Hsa_circ_0054633 knockdown reverses PDGF-BB-induced HA-VSMCs dysfunction. (A) qRT-PCR analysis of hsa_circ_0054633 expression in HA-VSMCs transfected with two forms of si-hsa_circ_0054633. HA-VSMCs were treated with PDGF-BB, PDGF-BB + si-NC, or PDGF-BB + si-circ\#1. After treatment, (B) qRTPCR analysis of hsa_circ_0054633 expression in HA-VSMCs; (C) CCK-8 assay of HA-VSMC proliferation; (D) flow cytometric analysis of cell cycle in HA-VSMCs; (E) transwell analysis of HA-VSMC migration; (F) ROS generation analysis of HAVSMCs with DCFH-DA assay; (G) western blot analysis of PCNA, cyclin D1, MMP9, and SOD2 expression in HA-VSMCs. $* P<0.05,{ }^{* *} P<0.01$.

Fig. 3 Hsa_circ_0054633 is a sponge of miR-107. (A) The putative binding sequences of miR-107 on hsa_circ_0054633. (B) qRT-PCR analysis of miR-107 expression in the plasma from CAD patients and healthy people. (C) qRT-PCR analysis of miR-107 expression in HA-VSMCs treated with or without PDGF-BB. (D) qRT-PCR analysis of miR-107 in HA-VSMCs transfected with miR-NC or miR-107. (E) Dual-luciferase reporter assay in HA-VSMCs co-transfected with hsa_circ_0054633-wt or hsa_circ_0054633-mut and the indicated miRNAs. (F) RIP assay for the enrichment of Ago2 on circ_0054633 and miR-107 in HA-VSMCs. (G) qRT-PCR analysis of hsa_circ_0054633 level in the materials pulled down by bio-miR-107. (H) qRT-PCR analysis of miR-107 level in HA-VSMCs transfected with si-NC or si-circ\#1. ${ }^{*} P<0.05$, $* * P<0.01$. 
Fig. 4 Hsa_circ_0054633 knockdown alleviates PDGF-BB-induced HA-VSMCs dysfunction via targeting miR-107. (A) qRT-PCR analysis of miR-107 level in HAVSMCs transfected with anti-NC or anti-miR-107. HA-VSMCs were transfected with si-NC, si-circ\#1, si-circ\#1 + anti-NC, or si-circ\#1 + anti-miR-107 after treatment with PDGF-BB. After transfection, (B) qRT-PCR analysis of miR-107 level in HA-VSMCs; (C) HA-VSMC migration analysis with transwell assay; (D) proliferation analysis of HA-VSMCs with CCK-8 assay; (E) cell cycle detection of HA-VSMCs with flow cytometry; (F) DCFH-DA assay of ROS generation in HA-VSMCs; (G) the detection of PCNA, cyclin D1, MMP9, and SOD2 expression in HA-VSMCs using western blot. ${ }^{*} P<0.05$.

Fig. 5 TXNIP is a target of miR-107. (A) The putative binding sequences of miR-107 on TXNIP 3'UTR. (B) qRT-PCR analysis of TXNIP expression in the plasma from CAD patients and healthy people. (C, D) TXNIP expression measurement in HAVSMCs treated with or without PDGF-BB using qRT-PCR and western blot. (E) Dualluciferase reporter assay in HA-VSMCs co-transfected with TXNIP 3'UTR-wt or -mut and miR-107 or miR-NC. (F, G) TXNIP expression detection in HA-VSMCs transfected with miR-NC or miR-107 using qRT-PCR and western blot. (H, I) TXNIP expression detection in HA-VSMCs transfected with si-NC, si-circ\#1, si-circ\#1 + anti$\mathrm{NC}$, or si-circ $\# 1+$ anti-miR-107 using qRT-PCR and western blot. $* P<0.05, * * P<0.01$. Fig. 6 Hsa_circ_0054633 moderates PDGF-BB-induced HA-VSMCs damage by regulating TXNIP. (A, B) TXNIP expression detection in HA-VSMCs transfected with vector or TXNIP with qRT-PCR and western blot. HA-VSMCs were transfected with 
595

596

597

598

599

600

601

602

603

604

605

606

607

608 609

si-NC, si-circ\#1, si-circ\#1 + vector, or si-circ\#1 + TXNIP after treatment with PDGFBB. After transfection, (C, D) TXNIP expression detection in HA-VSMCs using qRTPCR and western blot. (E) transwell assay of HA-VSMC migration; (F) ROS generation analysis of HA-VSMCs with DCFH-DA assay; (G) CCK-8 assay of proliferation in HA-VSMCs; (H) flow cytometric analysis of cell cycle in HA-VSMCs; (I) western blot analysis of PCNA, cyclin D1, MMP9, and SOD2 levels in HA-VSMCs. $\quad * P<0.05$, $* * P<0.01$.

Table 1. The clinical and demographic characteristics of the patients with CAD and Controls.

Table 2. Correlation of the expression of hsa_circ_0054633/miR-107/TXNIP with clinicopathologic features in patients of CAD 
Table 1. The clinical and demographic characteristics of the patients with CAD and Controls.

\begin{tabular}{cccc}
\hline & Normal(n=33) & CAD $(\mathrm{n}=33)$ & $P$ value \\
\hline Males/females & $15 / 18$ & $19 / 14$ & 0.33 \\
Age (years) & $56.1 \pm 5.6$ & $57.5 \pm 6.1$ & 0.34 \\
Body mass index $\left(\mathrm{kg} / \mathrm{m}^{2}\right)$ & $22.4 \pm 2.2$ & $23.8 \pm 2.5$ & 0.02 \\
Smokers \%(n) & $32.3(11)$ & $48.5(16)$ & 0.21 \\
Drinking \%(n) & $24.2(8)$ & $21.2(7)$ & 0.77 \\
Hypertension \%(n) & $12.1(4)$ & $60.6(20)$ & $<0.01$ \\
Diabetes mellitus \%(n) & $6.1(2)$ & $27.2(9)$ & 0.02 \\
Total cholesterol (mmol/L) & $4.3 \pm 1.1$ & $5.1 \pm 1.3$ & $<0.01$ \\
Triglyceride (mmol/L) & $1.3 \pm 0.4$ & $1.8 \pm 0.6$ & $<0.01$ \\
HDL-C(mmol/L) & $1.2 \pm 0.3$ & $1.1 \pm 0.4$ & 0.26 \\
LDL-C(mmol/L) & $2.9 \pm 0.8$ & $2.1 \pm 0.6$ & $<0.01$ \\
Hs-CRP (mg/L) & $1.6 \pm 0.7$ & $3.2 \pm 1.7$ & $<0.01$ \\
\hline
\end{tabular}

HDL-C, high-density lipoprotein cholesterol; LDL-C, low density lipoprotein Cholesterol; Hs-CRP: high-sensitivity C-reactive protein.

Data are represented as mean \pm SD or number (percentage) for category variables. $P$ : CAD vs. Normal 
Table 2. Correlation of the expression of hsa_circ_0054633/ miR-107/TXNIP with clinicopathologic features in patients of CAD

\begin{tabular}{|c|c|c|c|c|c|c|c|c|c|c|}
\hline \multirow{4}{*}{ Parameters } & & \multirow{2}{*}{\multicolumn{2}{|c|}{$\begin{array}{c}\text { hsa_circ_0054633 } \\
\text { expression }\end{array}$}} & & \multirow{2}{*}{\multicolumn{2}{|c|}{$\begin{array}{l}\text { miR-107 } \\
\text { expression }\end{array}$}} & \multirow{2}{*}{\multicolumn{3}{|c|}{$\begin{array}{c}\text { TXNIP } \\
\text { expression }\end{array}$}} & \multirow[b]{4}{*}{$p$-value } \\
\hline & & & & & & & & & & \\
\hline & \multirow[b]{2}{*}{$\mathrm{N}=33$} & High & Low & & High & Low & \multirow[b]{2}{*}{$p$-value } & \multirow{2}{*}{$\begin{array}{l}\text { High } \\
\mathrm{N}=17\end{array}$} & \multirow{2}{*}{$\begin{array}{l}\text { Low } \\
\mathrm{N}=16\end{array}$} & \\
\hline & & $\mathrm{N}=17$ & $\mathrm{~N}=16$ & $p$-value & $\mathrm{N}=17$ & $\mathrm{~N}=16$ & & & & \\
\hline \multicolumn{11}{|l|}{ Gender } \\
\hline Male & 19 & 9 & 10 & 0.58 & 10 & 9 & 0.88 & 8 & 11 & 0.21 \\
\hline Female & 14 & 8 & 6 & & 7 & 7 & & 9 & 5 & \\
\hline \multicolumn{11}{|l|}{ Age,years } \\
\hline$<60$ & 14 & 5 & 9 & 0.12 & 7 & 7 & 0.88 & 8 & 6 & 0.31 \\
\hline$\geq 60$ & 19 & 12 & 7 & & 10 & 9 & & 9 & 10 & \\
\hline
\end{tabular}

Smoking

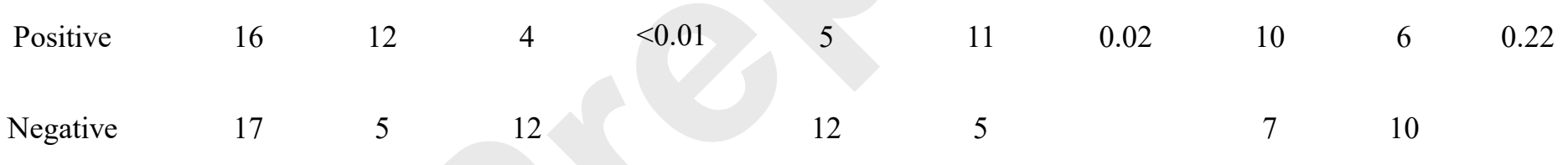

Drinking

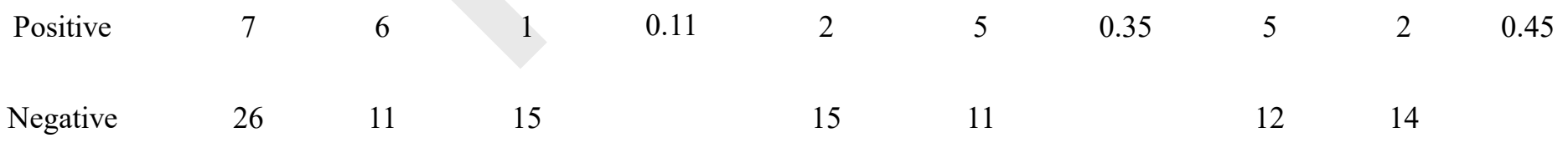

Hypertension

$\begin{array}{lcccccccccc}\text { Positive } & 20 & 14 & 6 & <0.01 & 7 & 13 & 0.02 & 15 & 5 & <0.01 \\ \text { Negative } & 13 & 3 & 10 & & 10 & 3 & & 2 & 11\end{array}$

Diabetes mellitus

\begin{tabular}{|c|c|c|c|c|c|c|c|c|c|}
\hline Positive & 9 & 8 & 1 & 0.03 & 2 & 7 & 0.10 & 7 & 2 \\
\hline Negative & 24 & 9 & 15 & & 15 & 9 & & 10 & 4 \\
\hline
\end{tabular}


Total cholesterol

(mmol/L)

$\begin{array}{llllllllll}\geq 5.1 & 16 & 13 & 3 & <0.01 & 5 & 11 & 0.02 & 14 & 2 \\ <5.1 & 17 & 4 & 13 & & 12 & 5 & & 3 & 14\end{array}$

\section{Triglyceride}

(mmol/L)

\begin{tabular}{|c|c|c|c|c|c|c|c|c|c|}
\hline$\geq 1.8$ & 15 & 12 & 3 & $<0.01$ & 4 & 11 & $<0.01$ & 13 & 2 \\
\hline$<1.8$ & 18 & 5 & 13 & & 13 & 5 & & 4 & 14 \\
\hline
\end{tabular}


A

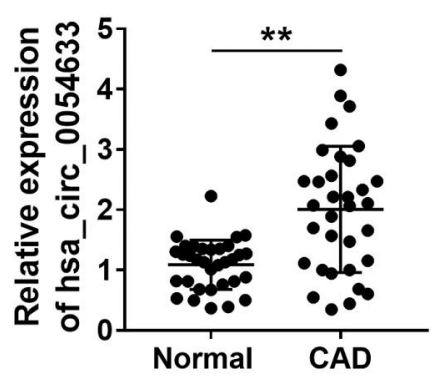

B

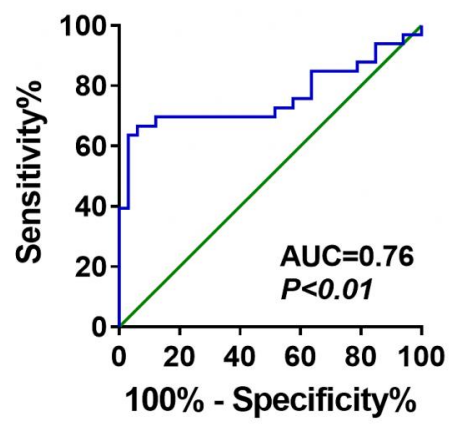

C

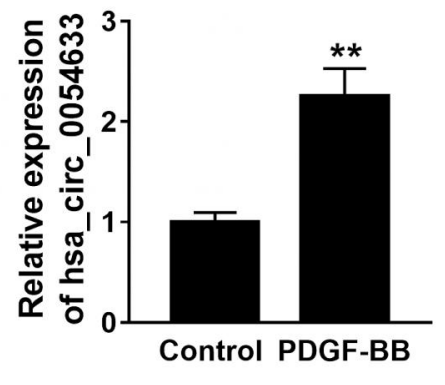

Fig. 1 Hsa_circ_0054633 expression is elevated in CAD patients and PDGF-BB-induced HAVSMCs. (A) The expression of hsa_circ_0054633 was detected in the plasma from CAD patients and healthy people using qRT-PCR. (B) ROC curves were plotted to determine the diagnostic value of hsa_circ_0054633 in plasma of CAD patients. (C) The expression of hsa_circ_0054633 was measured in HA-VSMCs treated with or without PDGF-BB by qRTPCR. ${ }^{*} \mathrm{P}<0.05,{ }^{* *} \mathrm{P}<0.01$. 
A

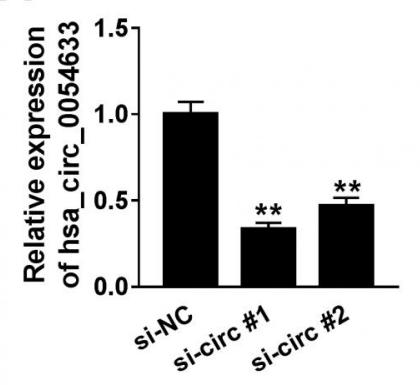

B

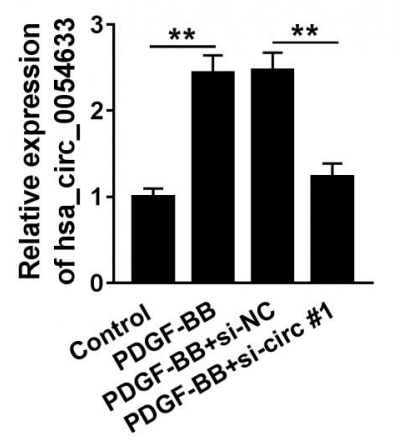

C

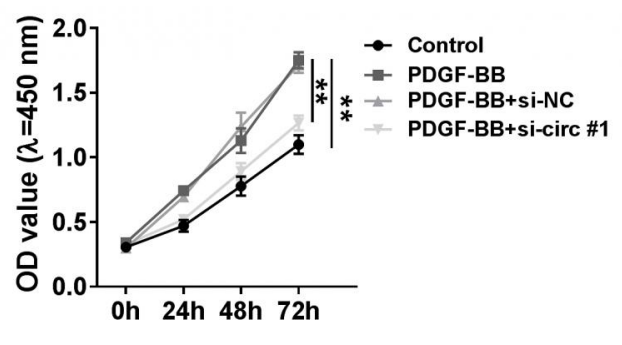

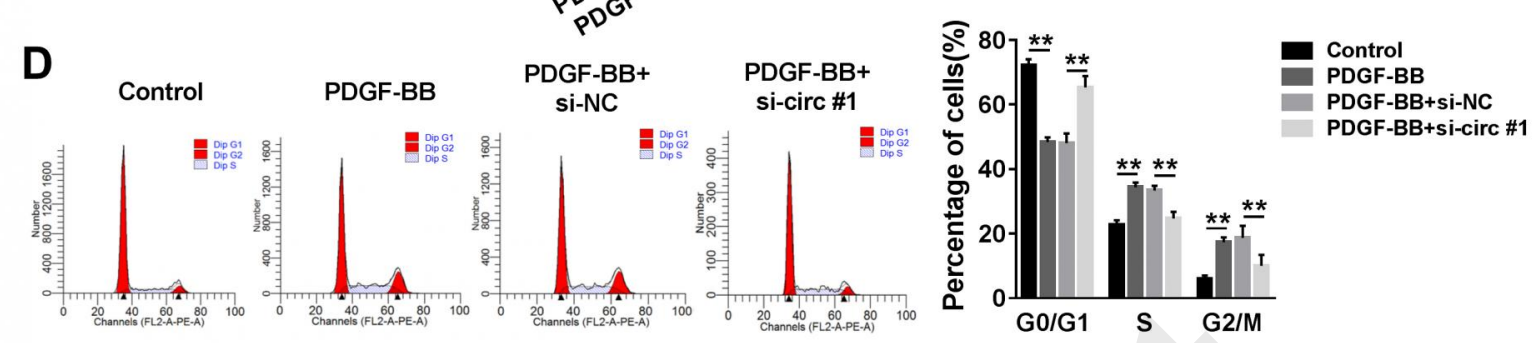

$\mathbf{E}$
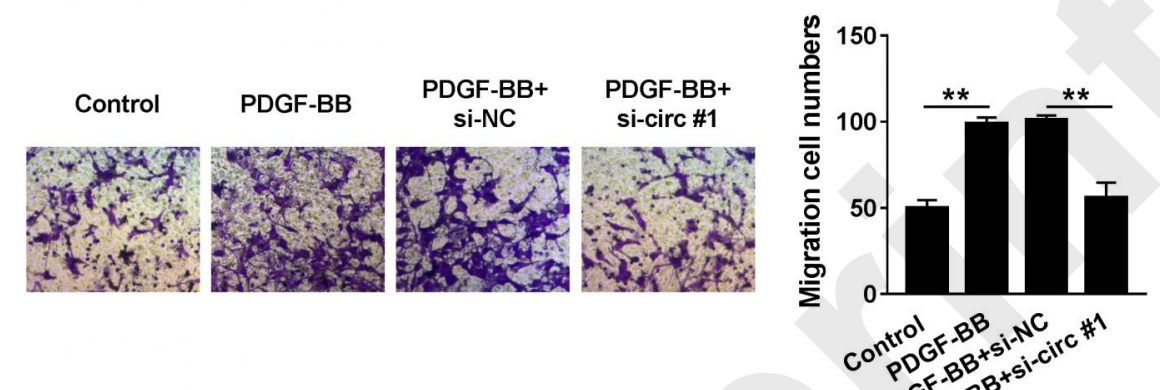

F

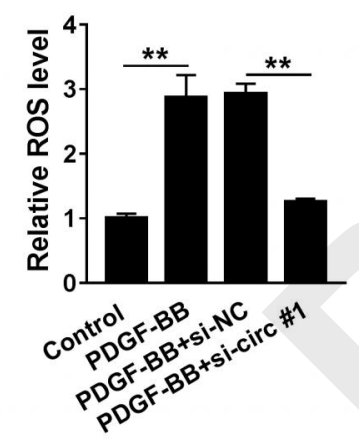

G

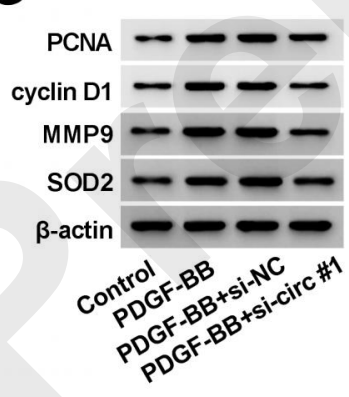

든

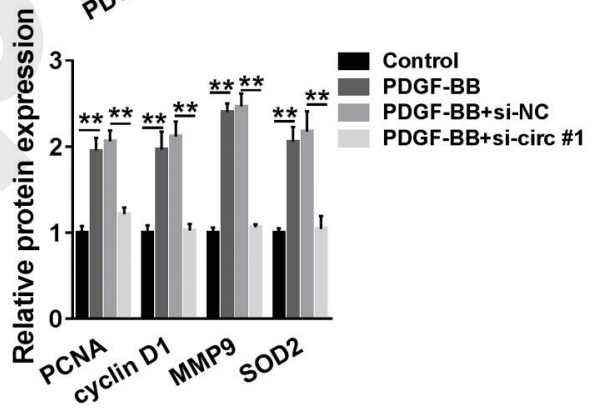

Fig. 2 Hsa_circ_0054633 knockdown reverses PDGF-BB-induced HA-VSMCs dysfunction. (A) qRT-PCR analysis of hsa_circ_0054633 expression in HA-VSMCs transfected with two forms of si-hsa_circ_0054633. HA-VSMCs were treated with PDGF-BB, PDGF-BB + si-NC, or PDGF-BB + si-circ\#1. After treatment, (B) qRT-PCR analysis of hsa_circ_0054633 expression in HA-VSMCs; (C) CCK-8 assay of HA-VSMC proliferation; (D) flow cytometric analysis of cell cycle in HA-VSMCs; (E) transwell analysis of HA-VSMC migration; (F) ROS generation analysis of HA-VSMCs with DCFH-DA assay; $(\mathrm{G})$ western blot analysis of PCNA, cyclin D1, MMP9, and SOD2 expression in HA-VSMCs. ${ }^{*} \mathrm{P}<0.05$, ${ }^{* *} \mathrm{P}<0.01$. 
A

Hsa_circ_00054633-mut

Hsa_circ_00054633-wt

miR-107

5' -AAAgAUAAAAAUUUCAUGCUGCA- 3'

| | || | |

3' -ACUAUCGgGaCAUGUUACGACGA- 5'
B

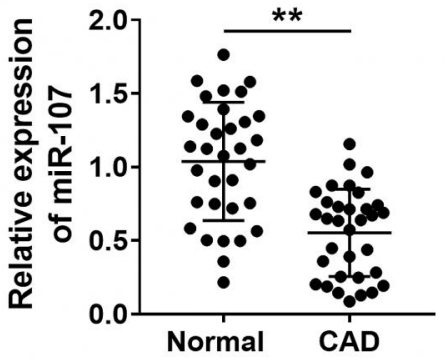

C

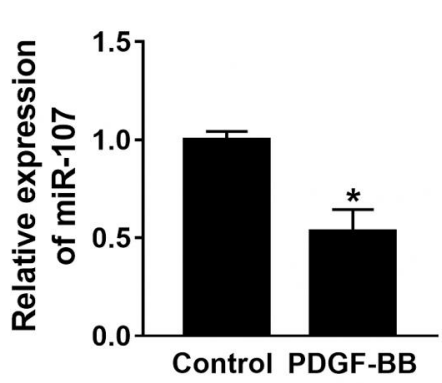

D

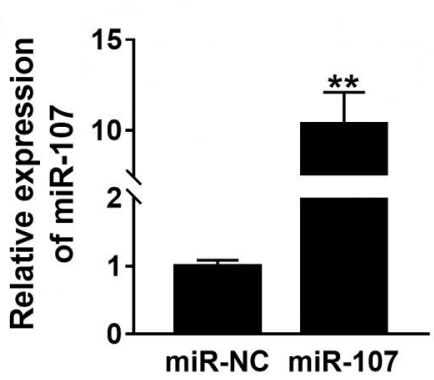

G

$\mathbf{F}$

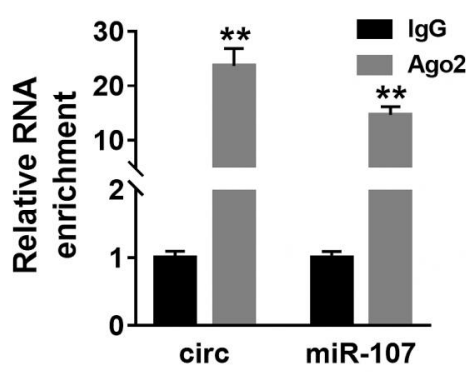

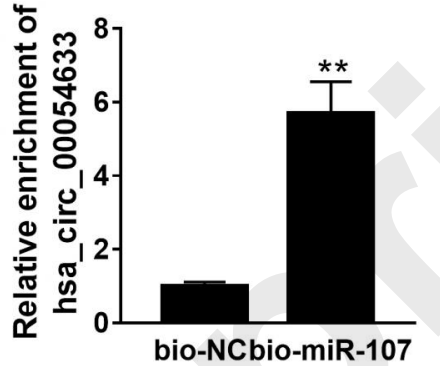

E

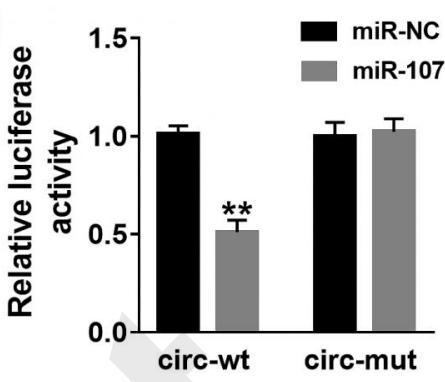

H

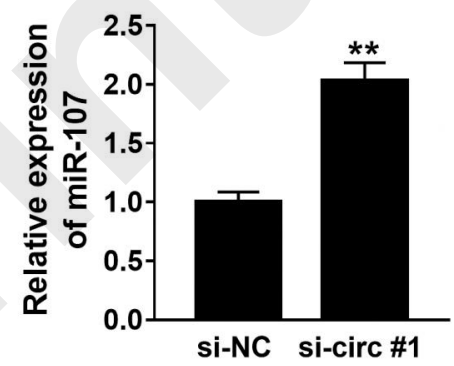

Fig. 3 Hsa_circ_0054633 is a sponge of miR-107. (A) The putative binding sequences of miR-107 on hsa_circ_0054633. (B) qRT-PCR analysis of miR-107 expression in the plasma from CAD patients and healthy people. (C) qRT-PCR analysis of miR-107 expression in HAVSMCs treated with or without PDGF-BB. (D) qRT-PCR analysis of miR-107 in HA-VSMCs transfected with miR-NC or miR-107. (E) Dual-luciferase reporter assay in HA-VSMCs cotransfected with hsa_circ_0054633-wt or hsa_circ_0054633-mut and the indicated miRNAs. (F) RIP assay for the enrichment of Ago2 on circ_0054633 and miR-107 in HA-VSMCs. (G) qRT-PCR analysis of hsa_circ_0054633 level in the materials pulled down by bio-miR-107. (H) qRT-PCR analysis of miR-107 level in HA-VSMCs transfected with si-NC or si-circ\#1. ${ }^{*} \mathrm{P}<0.05,{ }^{* *} \mathrm{P}<0.01$. 
A

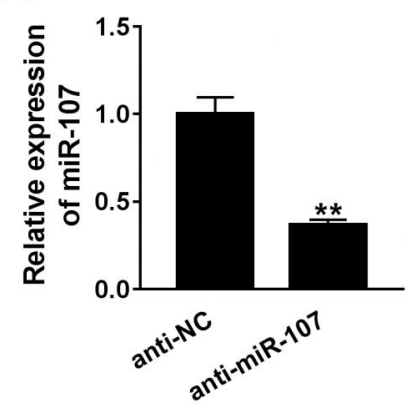

D

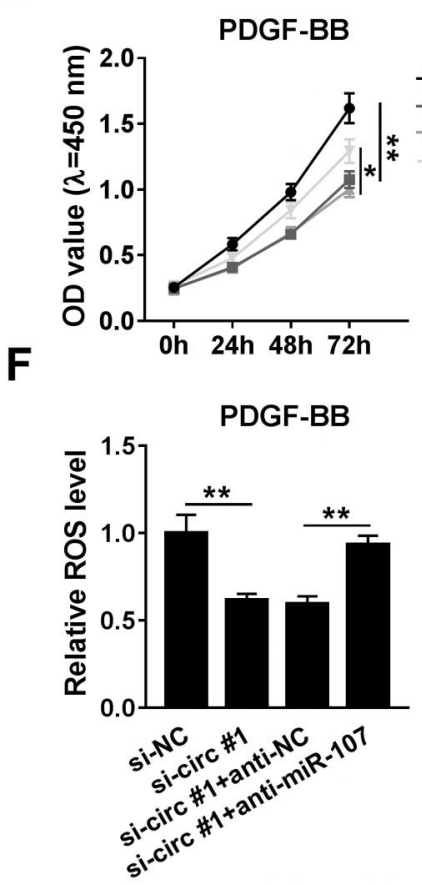

B

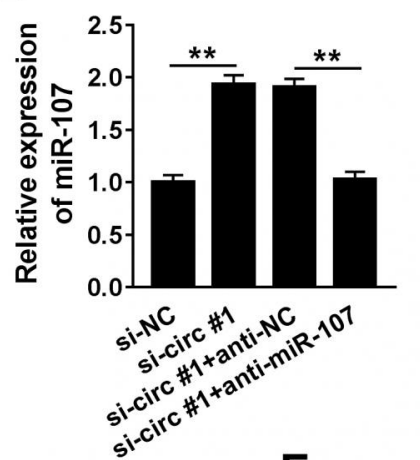

C

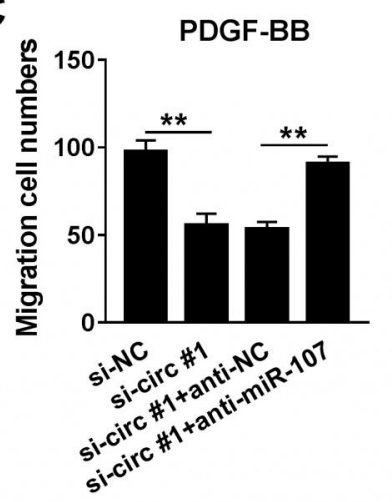

E

PDGF-BB

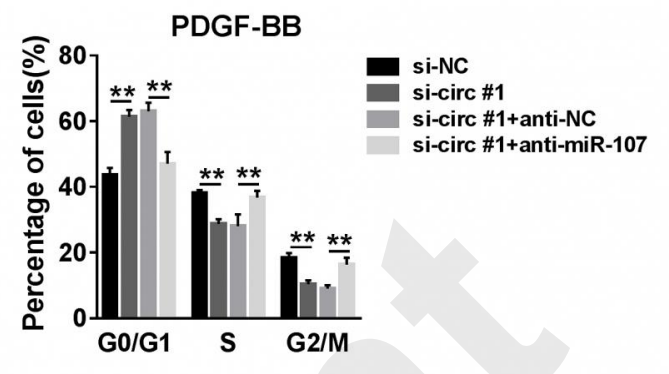

G
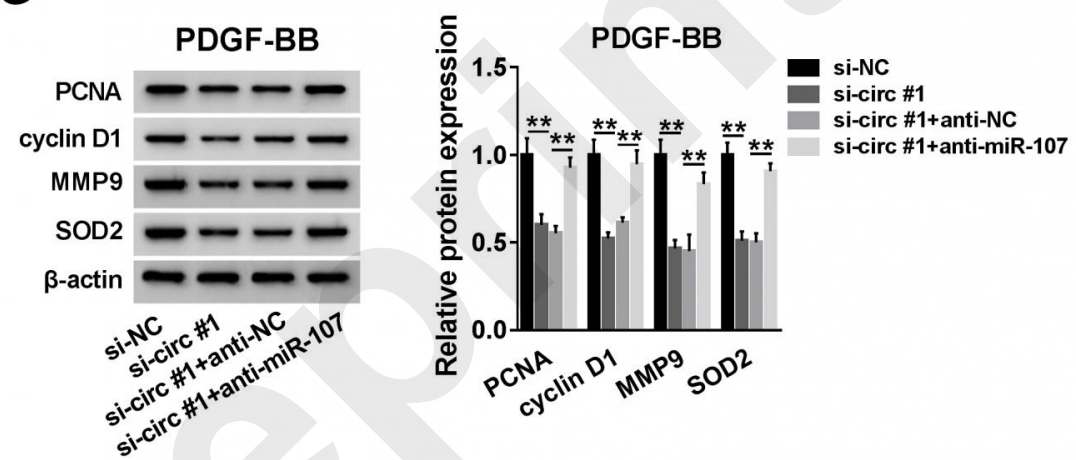

Fig. 4 Hsa_circ_0054633 knockdown alleviates PDGF-BB-induced HA-VSMCs dysfunction via targeting miR-107. (A) qRT-PCR analysis of miR-107 level in HA-VSMCs transfected with anti-NC or anti-miR-107. HA-VSMCs were transfected with si-NC, si-circ\#1, si-circ\#1 + anti-NC, or si-circ\#1 + anti-miR-107 after treatment with PDGF-BB. After transfection, (B) qRT-PCR analysis of miR-107 level in HA-VSMCs; (C) HA-VSMC migration analysis with transwell assay; (D) proliferation analysis of HA-VSMCs with CCK-8 assay; (E) cell cycle detection of HA-VSMCs with flow cytometry; (F) DCFH-DA assay of ROS generation in HAVSMCs; (G) the detection of PCNA, cyclin D1, MMP9, and SOD2 expression in HA-VSMCs using western blot. ${ }^{*} \mathrm{P}<0.05$. 
A

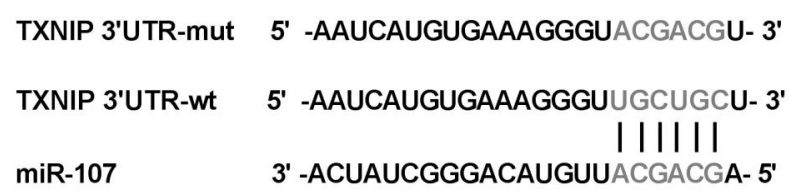

C

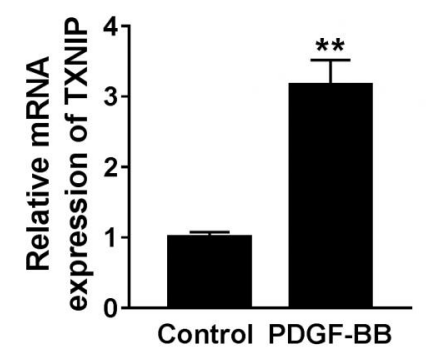

E

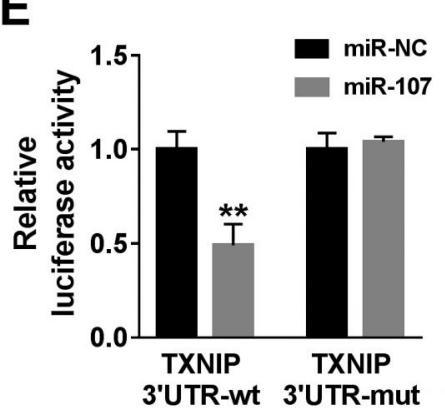

H

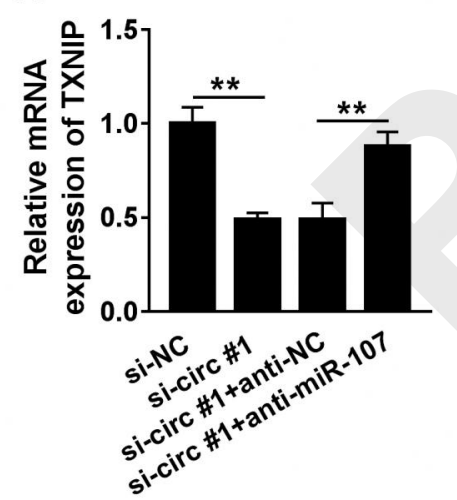

D

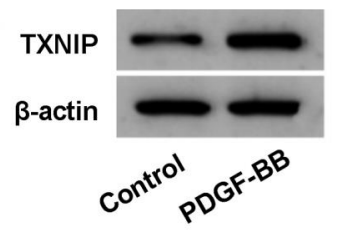

$\mathbf{F}$
B
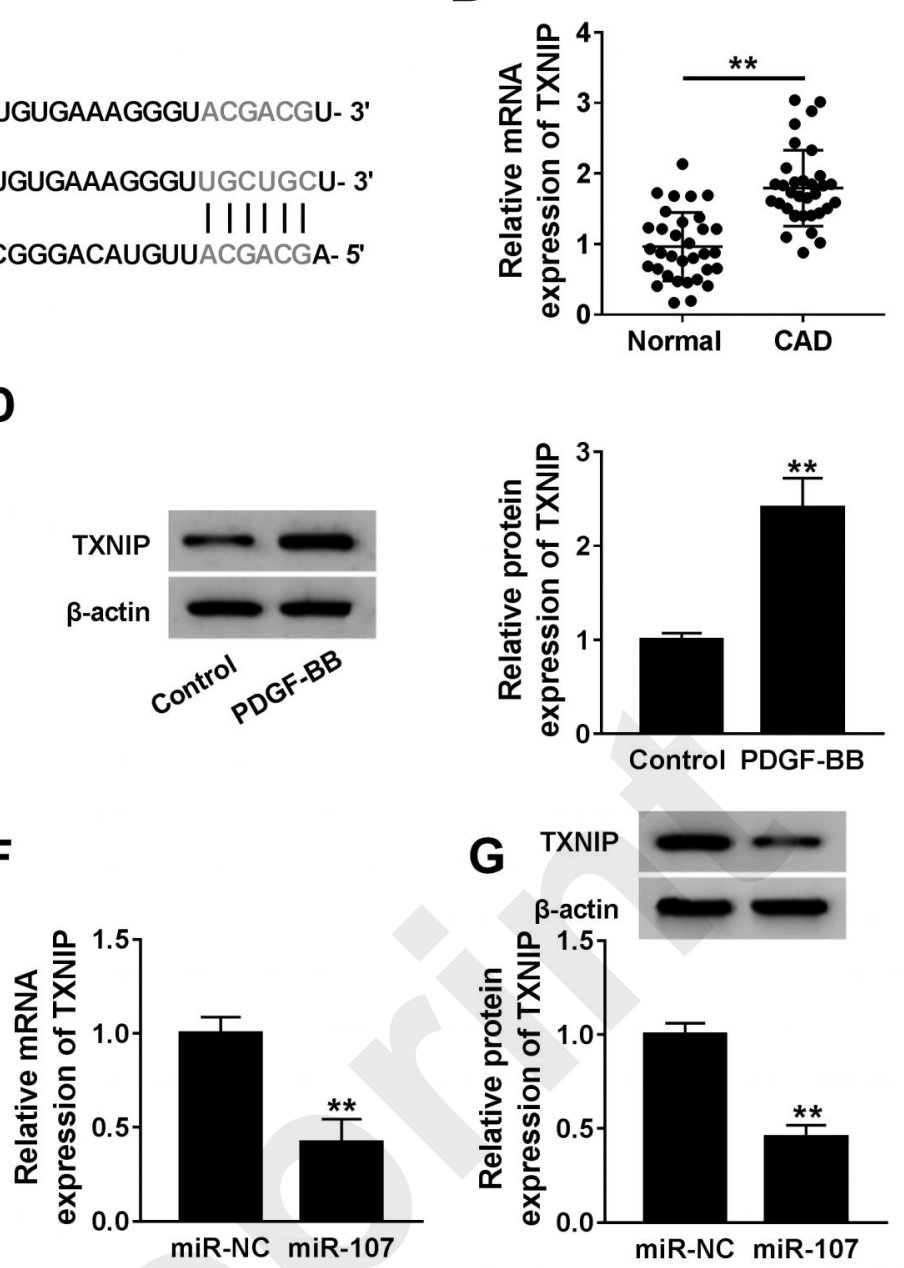

G
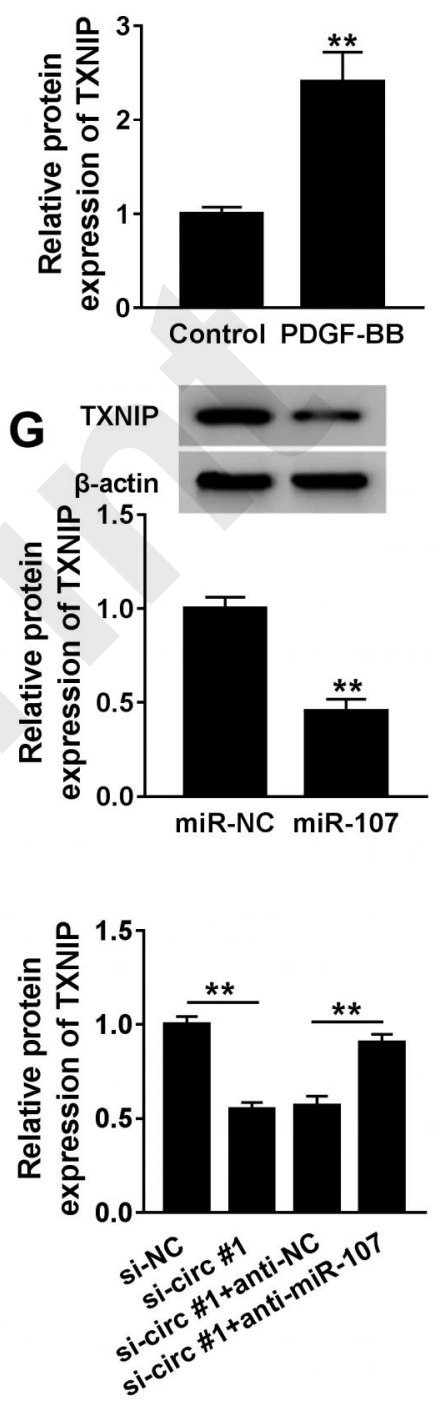

Fig. 5 TXNIP is a target of miR-107. (A) The putative binding sequences of miR-107 on TXNIP 3'UTR. (B) qRT-PCR analysis of TXNIP expression in the plasma from CAD patients and healthy people. (C, D) TXNIP expression measurement in HA-VSMCs treated with or without PDGF-BB using qRT-PCR and western blot. (E) Dual-luciferase reporter assay in HA-VSMCs co-transfected with TXNIP 3'UTR-wt or -mut and miR-107 or miR-NC. (F, G) TXNIP expression detection in HA-VSMCs transfected with miR-NC or miR-107 using qRTPCR and western blot. $(\mathrm{H}, \mathrm{I})$ TXNIP expression detection in HA-VSMCs transfected with siNC, si-circ\#1, si-circ\#1 + anti-NC, or si-circ\#1 + anti-miR-107 using qRT-PCR and western blot. ${ }^{*} \mathrm{P}<0.05,{ }^{* *} \mathrm{P}<0.01$. 
A
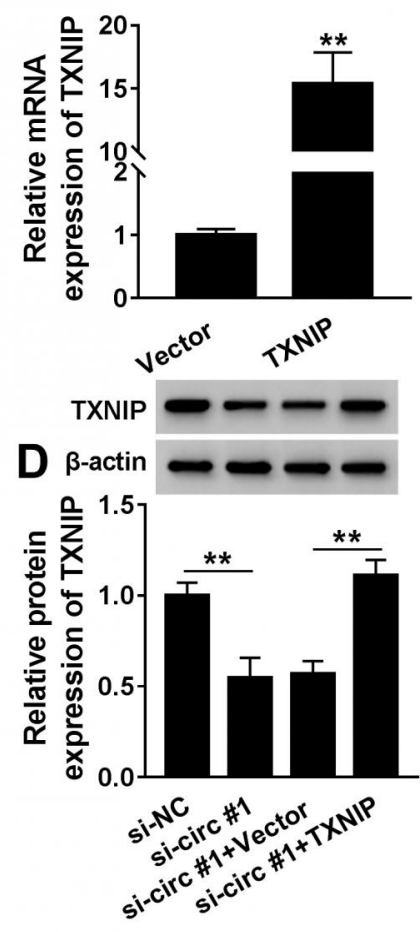

G

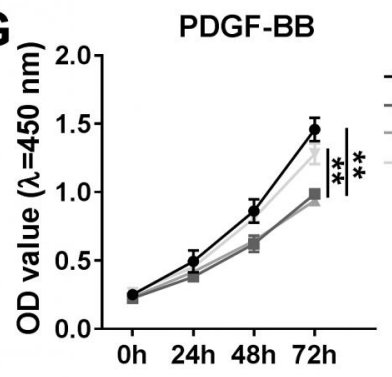

I

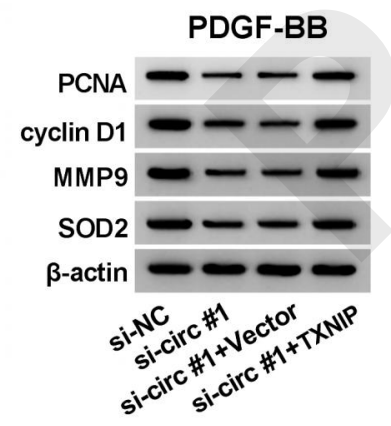

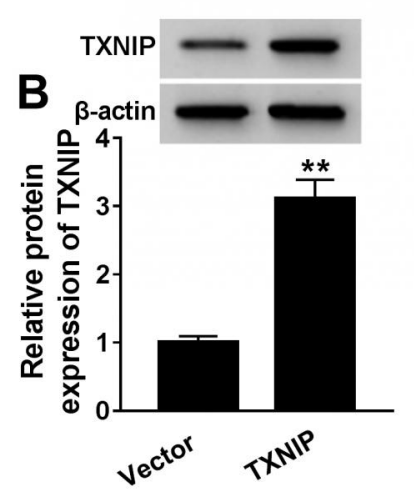

C
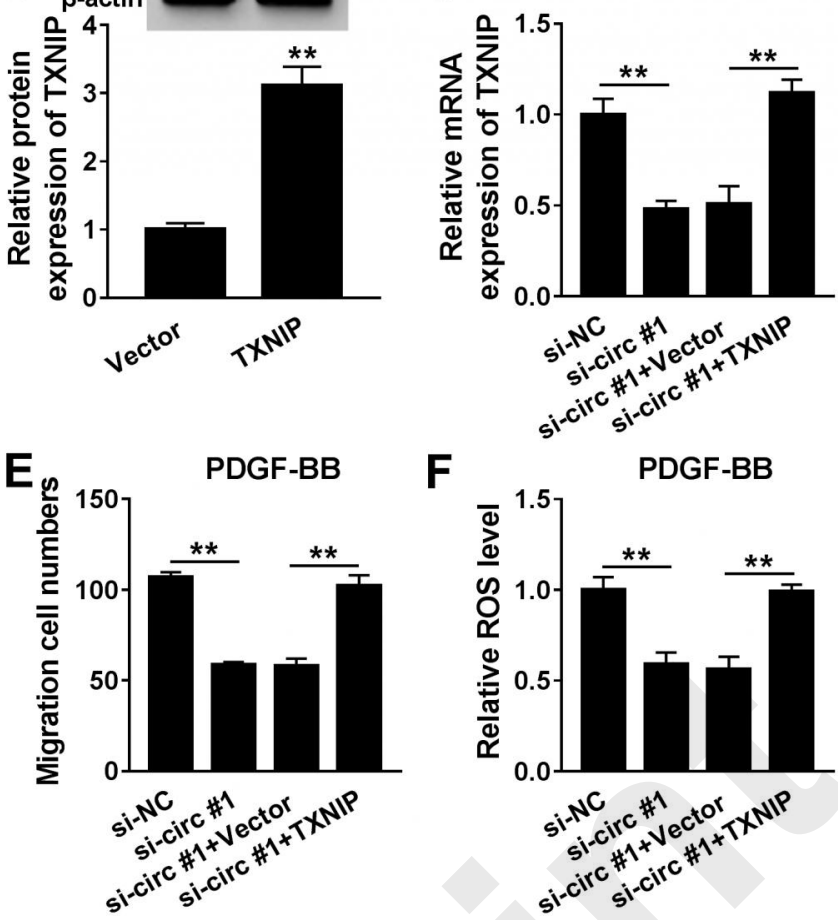

$\mathbf{F}$

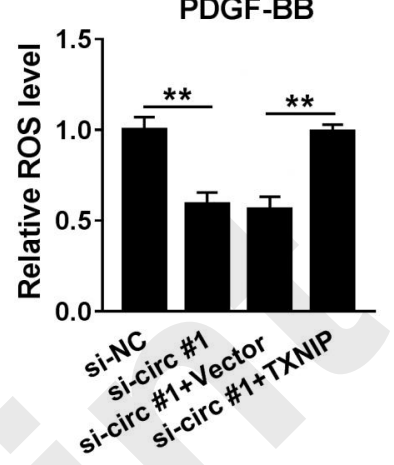

PDGF-BB
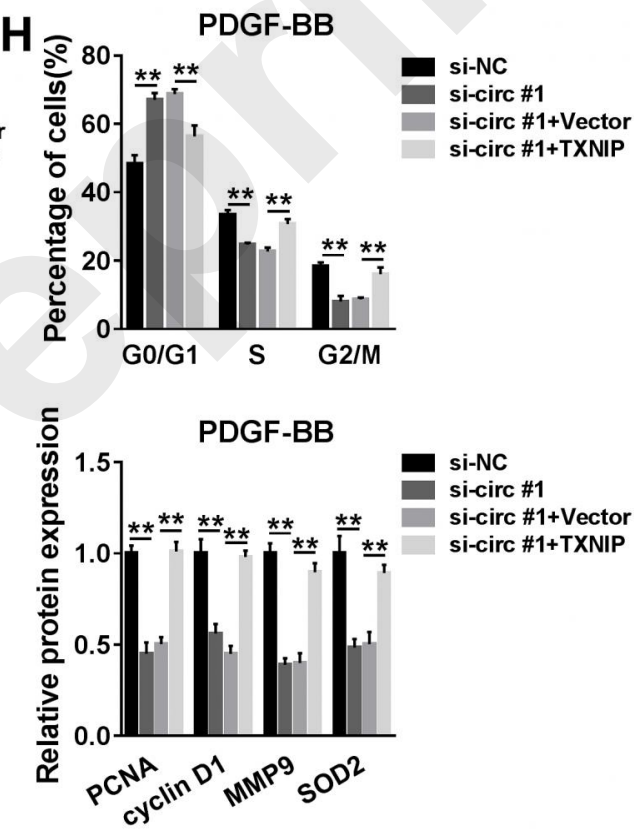

Fig. 6 Hsa_circ_0054633 moderates PDGF-BB-induced HA-VSMCs damage by regulating TXNIP. (A, B) TXNIP expression detection in HA-VSMCs transfected with vector or TXNIP with qRT-PCR and western blot. HA-VSMCs were transfected with si-NC, si-circ\#1, sicirc\#1 + vector, or si-circ\#1 + TXNIP after treatment with PDGF-BB. After transfection, (C, D) TXNIP expression detection in HA-VSMCs using qRT-PCR and western blot. (E) transwell assay of HA-VSMC migration; $(F)$ ROS generation analysis of HA-VSMCs with DCFH-DA assay; (G) CCK-8 assay of proliferation in HA-VSMCs; $(H)$ flow cytometric analysis of cell cycle in HA-VSMCs; (I) western blot analysis of PCNA, cyclin D1, MMP9, and SOD2 levels in HA-VSMCs. ${ }^{*} \mathrm{P}<0.05,{ }^{* *} \mathrm{P}<0.01$. 